\title{
Skin Graft - General Considerations
}

\section{Sundaram Rajaram* and Gauthami Sundar}

University of Science and Technology, Fujairah, United Arab Emirates

*Corresponding Author: Sundaram Rajaram, University of Science and

Technology, Fujairah, United Arab Emirates.
Received: October 20, 2021

Published: November 16, 2021

(C) All rights are reserved by Sundaram

Rajaram and Gauthami Sundar.

\begin{abstract}
The procedure of skin grafting is an age old technique. Many new developments have happened in the armamentarium of skin grafting which is aimed at producing better aesthetic results and reducing complications. Although the skin grafting has seen new advancements, the basic principle remains unaltered. The presence of a healthy vascular recipient bed is an important factor for the graft survival. Skin graft still remains an important option in the reconstructive ladder.
\end{abstract}

Keywords: Split Thickness Skin Grafts; Full Thickness Skin Grafts; Skin Graft Harvesting; Skin Graft Fixation; Graft Healing

\section{Introduction}

Wound healing is one of the most eventful phenomena seen in physiology. Wounds healing with primary intention heal the best with minimal complications. Wounds that heal otherwise (secondary intention) are bound to have added complications that can happen like infection, scarring, scar contracture. prevention of such issues are done by harvesting grafts or flaps to cover up such defects in order to promote primary intention wound healing thereby wound heals the best. Skin Grafts become the first line choice of covering such defects. Since time immemorial, skin grafts had been practiced and have been identified with many parts of the globe at varied timelines. This chapter elaborates the armamentarium and techniques involved in skin grafting and methods of fixation.

\section{History of skin grafting and armamentarium}

The history of free skin grafting dates back to as early as $600 \mathrm{BC}$ in ancient India where the defects of the ears nose and lips were treated using free gluteal grafts and skin grafts [1].

Tile maker caste have been known for practising free skin grafts, harvested from the gluteal region which was prepared by beating with wooden slippers until significant swelling had taken place they also used a secret cement for adhesion of the skin grafts which was called the "ancient Indian method" [2].
After a long stint of time it was G. Baronio in the $18^{\text {th }}$ century who documented the use of skin grafting so his skin grafting techniques involved tail of a sheep as a donor sites. All the transplanted skin grafts on the same animal were found to be remarkably successful [2]. Bunger in 1823, inspired by "Ancient Indian Method" harvested free whole thickness skin grafts from the thigh for covering the nasal defects in which he found moderate success. Sir Astley cooper transplanted the skin from the amputed thumb for covering the stump defect [3].

Reverdin in $19^{\text {th }}$ century introduced "Epidermic grafts" which was popularly called as "the pinch grafts". In this technique the skin was pinched up and cut off and were placed on the healing granulating wounds, there by covering the wound. Although the pinch graft were very popular cosmetically it gives very poor results [5].

Following Reverdin Oilier in the late $19^{\text {th }}$ century transplanted much larger films of skin and found success in it. Carl Thiersch (in 1886) presented a method of skin grafting which involved large films of epidermis with less dermal components which is the same as oilier. Later this technique was named as Oilier-Thiersch graft and also has been called the "split graft".

In 1875 John Reissberg Wolfe of Glasgow successfully covered a lower eyelid defect transplanted from the arm. Since the introduc-

Citation: Sundaram Rajaram and Gauthami Sundar. "Skin Graft - General Considerations". Acta Scientific Dental Sciences 5.12 (2021): $37-44$. 
tion of Oilier Thiersch grafts and many other surgeons, practising skin harvesting with differential thickness, leading to better donor site healing the need for better sophisticated instruments found its rise [6].

Blair knife, Catlin knife and Ferris - Smith knife were the simplest of knifes that were used to harvest split thickness skin grafts in the initial days [7].

Vilray Papin Blair in 1930 tactfully used the suction apparatus to place the skin under tension and used a knife to harvest skin grafts [6]. John Staige David in 1909 presented a detailed account of using a Catlin knife in harvesting skin grafts. Hofmann developed a guarded knife which permitted him to adjust the depth of the skin to be sliced which was regulated by screws. However Ricardo Finochietto, invented a revise version of Hoffmans knife which became very popular [9]. So henceforth, regulating the thickness of skin grafts became the order of practice.

Graham Humby in England was the first to use a depth control knife in harvesting skin grafts. He added roller to the Blair knife thereby making it modified. The distance between the both was adjustable by means of a calibration device [10]. Humby later modified his device in which the tension on the skin was created using tiny needles on a cross bar which pierce the skin to depth of oneeighth of an inch. It was having another frame work in which is a knife with an adjustable rolling and sling guard. This device was mainly used on the limbs [11]. However this technique was very combersone because of the size of the frames used. So Humby discarded the large frame and used only a solid blade. The knife had a rod which is connected to the upper surface with scres that allow it to control the depth [12].

Denis Charlis Vodenham introduced replaceable blades thus avoiding the frequent disadvantage of sharpening the blade and it also enjoys the advantage of having a better hygiene and minimal drag from bluntness $[13,14]$. Braithwaite Fenton in 1955 introduced the knife in which the blade need not be dismantled [15]. But Braith Waite Knife has a tendency to make the skin rolled up during harvest. This required an assistant to hold the free ends of the skin graft while being harvested. It can also lead to uneven thickness of the harvested skin and in some cases the skin grafts can also get cut in the midway [16] all this disadvantages were owed to the roller that slide to and fro along its long axis. Watson john in 1960 identified the problem and introduced a knife that had a rod with no independent movement [17] the drag that used to be seen while using these knifes could be avoided by lubricating the skin surface using liquid paraffin [18]. Cobbett made a modification to Braithwaite knife by cutting a' D' section instead of a circular hole in the guard end. This was done to avoid the drag and rolling up of the skin [16].

Goullian used straight razor with a handle that can cut different thickness of the skin [19] snow used Shick injector razor in harvesting skin grafts [20]. Shoul modified a Gillette safety razor for harvesting skin grafts [21] silver in 1959 also joins the list of people who used razors for skin grafting [22-24] in 1939 Dr Earl Padgett invented a device which was based on the adhesion-traction principle named as dermatomes following him harry M. brown in vented an electric dermatome. There were further modification in the dermatome like the Pagette dermatome, Reese dermatome, the brown dermatome, the Stryker dermatome and the Castroviejo [25-27].

\section{General considerations}

The general plan of the surgery is to be considered at the inception. The requirement of the type, dimension and access of the defect determines the type of the skin graft (sheets or pinch graft), the thickness of the graft (split or full thickness) and the inclusion of special components (composite grafts).

Based on the types, it can be classified as follows.

- Mesh grafts

- Sheet grafts

- $\quad$ Pinch grafts.

Based on the thickness, the skin grafts are classified as mentioned in the table (Table 1).

\begin{tabular}{|l|c|c|}
\hline S no & Name of the graft & Thickness \\
\hline 1 & Thin split thickness (Thiersch-Ollier) & $0.15-0.3$ \\
\hline 2 & Intermediate split thickness (Blair-Brown) & $0.3-0.45$ \\
\hline 3 & Thick split thickness (Padgett) & $0.45-0.6$ \\
\hline 4 & Full thickness (Wolfe-Krause) & $>0.6$ \\
\hline
\end{tabular}

Table 1

Preparation of the recipient bed makes the perfect atmosphere for the 'graft take'. It needs to be devoid of any bleeding and debris and infection which would promote to the success of the graft healing [28]. 


\section{Surgical anatomy of the skin}

The skin is composed of 2 layers predominantly, namely the dermis (95\%) and epidermis (5\%). The thickness of the skin varies at varying age groups and varying anatomic locations. The vasculature to the skin runs parallel to the dermis and subdivide as the cutaneous vessels which run at right angles to penetrate the subcutaneous tissue and terminate between the dental papillae. Besides, the dermis also houses the sweat glands, sebaceous glands, subcutaneous fat and hair follicles [29] (Figure 1).

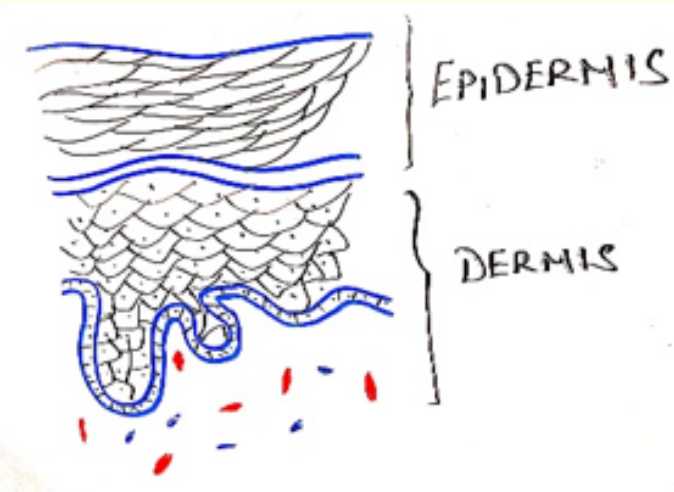

Figure 1

Surgical Procedure:

Full thickness skin grafts: (Figure 5 a, b, c)

- Preparation of donor site is done with povidone iodine solution.

- A pinch test is done on the donor site skin to know the level of plasticity (Figure 2).

- A template made out of a sterile paper to know the dimensions of the skin graft to be harvested.

- Local anaesthetic is infiltrated subcutaneously (Figure 3).

- Incision is placed along the resting skin tension lines in an elliptical fashion so that primary wound closure of the donor site can be done (Figure 4).

- Subdermal blunt dissection is carried out using scissors or finger pressure.

- Haemostasis achieved.

- The edges are undermined to allow tension free closure

- Double layer closure carried out.

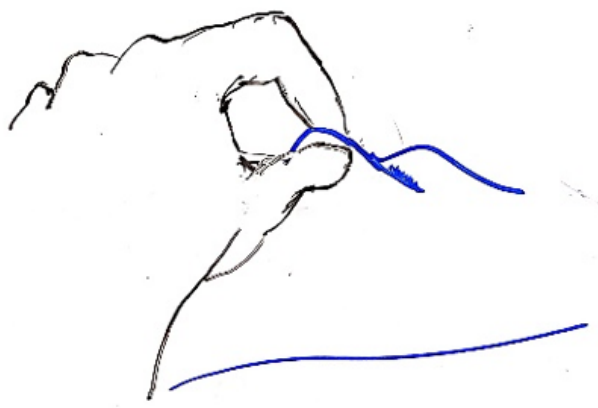

Figure 2

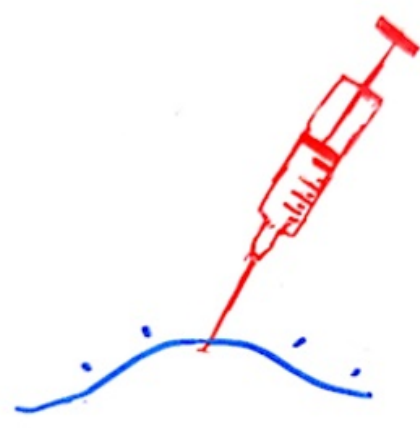

Figure 3

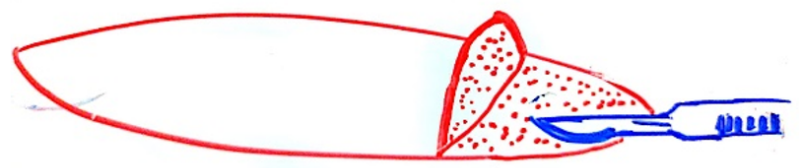

Figure 4

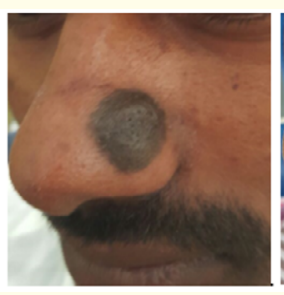

(a)

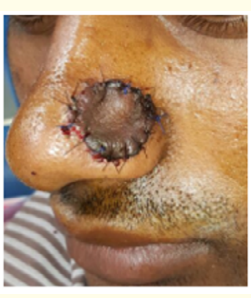

(b)

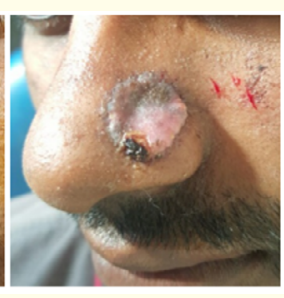

(c)
Figure 5 
Split thickness skin grafts: (Figure 6)

- Bevelling of the wound edges at the recipient site to allow the skin graft to blend at the edges. Haemostasis achieved. A template is prepared to know the dimensions of the defect. The template is done $20 \%$ larger than the defect size so that the wound contracture would be compensated.

- Preparation of donor site is done with povidone iodine solution. Mineral oil is applied to moisten the skin.

- The dermatome is applied over the prepared skin and settings are done with the dermatome to achieve the desired level of skin thickness (0.012 inch). It is placed to the skin at 45 degree angulation

- The skin tension is created by the an assistant who can make the surface flat

- Incision is placed along the resting skin tension lines in an elliptical fashion so that primary wound closure of the donor site can be done

- Once the desired length of skin graft harvest is achieved, the dermatome is angled away and the distal edge of the skin is cut using a scalpel

- $\quad$ The graft is transferred to a sterile plastic tray and small incisions of $1 \mathrm{~mm}$ are placed (pie crusting).

- Meshing can also be done to expand the skin graft to cover the defect with recommended ratios $(1.5: 1,2: 1,3: 1)$

- $\quad$ The graft needs to be protected with saline soaked gauze until it is transferred to the recipient site.

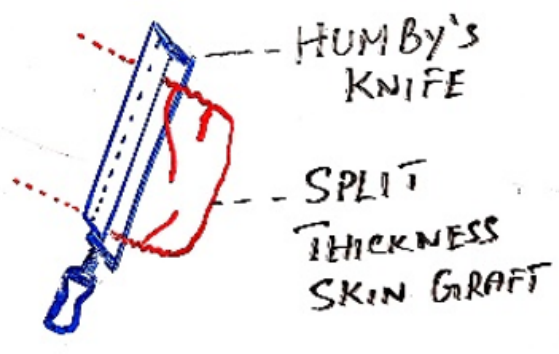

Figure 6

\section{Fixation of skin grafts}

- $\quad$ The skin edges are trimmed and the recipient wound edges are undermined to accommodate the skin graft. The edges of the skin graft are approximated and secured to the edges of the recipient wound with sutures or staples. Appropriate dressing is placed over the skin graft for better adaptation and graft healing. This also avoids the seroma formation and hematoma formation that can subsequently lead to infection and graft failure.

- Netscher and associates advocate moist non adherent gauze applied over the grafted site and is secured with self-adhering foam. Application and removal of the dressings are technically easy.

- $\quad$ Saltz and Bowles also advocate using Reston foam applied over Xenoform gauze as graft dressings.

- Balakrishnan advocates the use of Lyofoam, which is applied over the graft directly. It is a biologically inert substance and bacteriostatic.

- Johnson, flemming and Avery used staples with latex foam [30-33].

Tie over dressing/bolster technique: (Figure 7)

- The tie over dressing is one of the earliest and effective methods for graft fixation. Once the graft is transferred to the recipient bed and secured with sutures, a bolster is placed on the skin graft and secured with silk sutures running over the bolster and offering some pressure that prevents dislodgement. The bolster generally would be a piled up gauze pieces.

- Although supported only by some observational studies, the tie over dressing remains simple and effective means for skin graft fixation.

- Tie over dressing involves downward pressure on the skin graft surface thereby adapting the skin graft well onto the recipient area thus eliminating the hematoma and seroma formation leading to good take of the skin graft. The principle of tie over dressing remains as simple as that.

- $\quad$ Such a simple technique also is accountable for flipside issues inviting criticism. The downward pressure when it exceeds the capillary pressure, can cause graft damage. Prolonged intraoperative time and graft healing time, technique sensitive procedure, may hinder inspection and wound care in the postoperative period are other disadvantages. Also, no Randomized Control trials exist to prove the superiority of tie over technique over non tie over techniques [34]. 


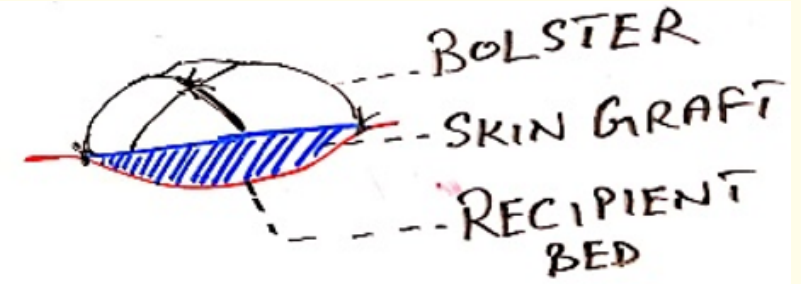

Figure 7

\section{Quilting: (Figure 8)}

- Quilting involves placing basing sutures on the surface of the graft thereby adapting it well to the recipient bed. Such quilting sutures are generally placed using absorbable ones.

- Quilting technique cannot be done in all the areas of the body like the nasal ala where the anatomy of the underlying nasal cartilage does not permit the placement of sutures [34].

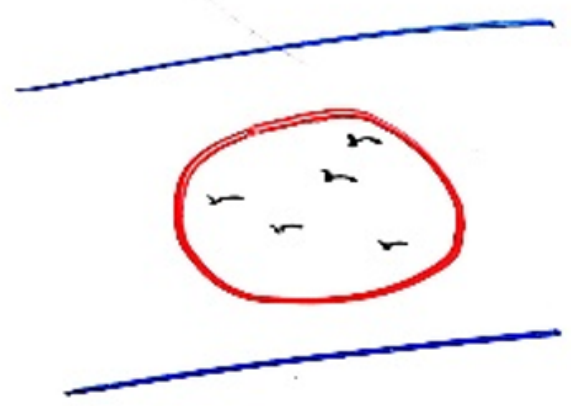

Figure 8

Tie over dressing vs no tie over technique

Akhavani., et al. and Dhillon., et al. compared both these techniques to find out there is no statistically significant difference in graft take rate and infections. Even a study conducted by Yuki., et al. in 266 patients also concluded the same [35,36].

\section{Other techniques}

- $\quad$ Modern techniques involve use of fibrin glue, super glue which are tough transparent materials that can adhere well with the tissue surfaces and are bio friendly. Negative pressure dress- ings can be useful for graft take where bolsters cannot be applied E.g.: axilla.

- $\quad$ Acrylic stents can be placed in the palatal grafts to support the graft take. The stent can be fixed using stainless steel wires [37-42] (Figure $9 \mathrm{a}, \mathrm{b}$ ).

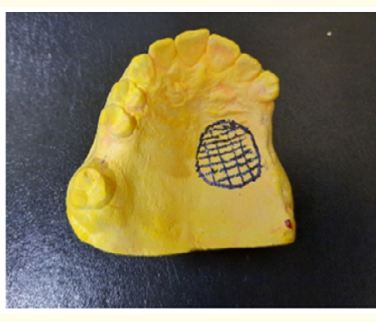

(a)

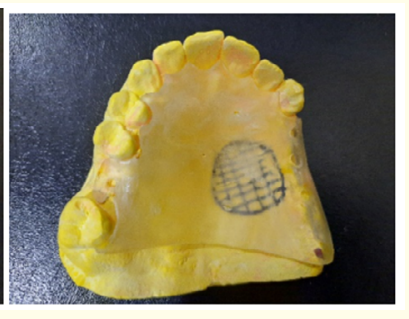

(b)
Figure 9

\section{Skin graft healing}

The graft healing is also known as 'Graft Take'.

Healing of graft happens through 3 stages.

- $\quad$ Stage I- Plasmatic imbibition

- $\quad$ Stage II- Inosculatory phase

- $\quad$ Stage III- capillary ingrowth.

\section{Plasmatic imbibition}

This initial phase lasts for 24-48 hours. In this phase the graft is completely shut off from blood. The ischemic status of the graft would extend to a maximum of 24 hours. Greater the ischemic time greater are the chances for failure. Although documentation reveals that full thickness grafts can survive the ischemic phase upto 3 days and split thickness grafts for upto 5 days, the exact ischemic tolerance level is unclear.

During this phase, the plasmatic circulation provides nourishment leading to weight gain of the graft [43-52].

\section{Inosculatory phase}

At the end of 48hours, the capillary growth is seen at the graft interface trying to vascularize the graft. This is clinically seen as the pinkish color change of the skin graft. 
Capillary ingrowth

- Capillary revascularization happens by two mechanisms - primary revascularization and secondary revascularization.

- Primary mechanism involves direct anastomosis of the capillary proliferation from the recipient bed and neovessels in the skin graft, thus leading to revascularization of the graft.

- Secondary revascularization involves production of vasoactive substances during the ischemic period as it is induced by ischemia [53].

\section{Conclusion}

Skin graft is a reconstructive technique that has been in practice for a long time in history. The technique has a simple physiology which remains unaltered till date. The techniques involved in skin graft harvesting has seen a gradual evolution leading to the modern day devices of effective skin graft harvesting. Many methods of fixation of the skin grafts are available and the suitable one is dictated by the situation of the defect size and location. It is these techniques of harvest and fixation that determine the success of the graft other than the systemic factors. Any surgeon should aim at perfecting these techniques for achieving best results.

\section{Acknowledgments}

Dr. Manjunath N.M MDS,M Phil, FAM, FAOCMF Consultany Oral and Maxillofacial Surgeon, Director of Dew Dental and maxillofacial Center, Bangalore, Karnataka.

\section{Conflict of Interest}

The authors declare no conflict of interest.

\section{Notes/Thanks/Other Declarations}

I extend my sincere thanks and gratitude to Dr. Gauthami Sundar and Dr. Rajaram for the timely support.

\section{Bibliography}

1. Chick LR. "Brief history and biology of skin grafting". Annals of Plastic Surgery 21 (1988): 358-365.

2. Davis JS. Address of the President: "The Story of Plastic Surgery". Annals of Surgery 113 (1941): 641-656.

3. Tiersch C. "Über Hautverplanzung". Verh Dtsch Ges Chir (1886): 15-17.
4. Gerrie JW. "The Choice of Skin Grafts in Plastic Surgery". Canadian Medical Association Journal 44 (1941): 9-13.

5. Converse JM. "Early skin grafting in war wounds of the extremities". Annals of Surgery 115 (1942): 321-335.

6. Converse JM and BauerRO. Transplantation of Skin. In: Converse JM, editor. "Reconstructive Plastic Surgery: Principles and Procedures in Correction, Reconstruction and Transplantation". $1^{\text {st }}$ edition. Philadelphia: W. B. Saunders (1964): 26-32.

7. Davis JS. "Plastic Surgery Its Principles and Practice". 1st edition. Philadelphia: P. Blakiston's and Son (1919).

8. Davis JS. "Skin Grafting at the Johns Hopkins Hospital. Discussion on Modern Methods of Skin Grafting". Annals of Surgery 50 (1909): 542-549.

9. Converse JM. "Grafts of Skin and Mucosa”. In: Converse JM, editor. Kazanjian and Converse's surgical treatment of facial injuries. 3rd ed. Baltimore: Williams and Wilkins (1974): 531-554.

10. Clarkson P. "The Humby". British Medical Journal 2 (1952): 1101.

11. Humby G. "Apparatus for Skin Grafting". British Medical Journal 1 (1934): 1078.

12. Humby G. "Modified Graft Cutting Razor". British Medical Journal 2 (1936): 1086.

13. Bodenham DC. "A new type of knife for cutting skin grafts, using replaceable blades". British Journal of Plastic Surgery 2 (1949): 136.

14. Bodenham DC. "A new type of knife for cutting skin grafts, using replaceable blades". Plastic and Reconstructive Surgery 5 (1950): 108.

15. BraithwaiteF. "Modification of Humby knife". Lancet 268 (1955): 1004.

16. Cobbett J. "A modification of the Braithwaite knife". British Journal of Plastic Surgery 21 (1968): 216-217.

17. Watson J. “A skin-grafting knife”. Lancet 2 (1960): 687-688.

18. Brown JB and Lee WE. "The repair of surface defects of the hand Skin grafting”. Annals of Surgery 107 (1983): 952-71.

19. Goulian D Jr. "Anew economical dermatome". Plastic and Reconstructive Surgery 42 (1968): 85-86. 
20. Snow JW. "Safety razor dermatome". Plastic and Reconstructive Surgery 41 (1968): 184.

21. Shoul MI. "Skin grafting under local anesthesia using a new safety razor dermatome". The American Journal of Surgery 112 (1966): 959-963.

22. Silver HL. "A new dermatome". Canadian Medical Association Journal 80.10 (1959): 828.

23. Silver HL and Gerrie JW. "A new dermatome: Discussion on modern methods of skin grafting, the choice of skin grafts in plastic surgery". Canadian Medical Association Journal 80 (1959): 828.

24. Silver HL and Kilner TP. "A new dermatome: The thiersch graft". its preparation and uses. Canadian Medical Association Journal 80 (1959): 828.

25. Bennett JE and Miller SR. "Evolution of the electro-dermatome". Plastic and Reconstructive Surgery 45 (1970): 131-134.

26. Stark RB. "John Davies Reese and the Reese dermatome". Annals of Plastic Surgery 2 (1979): 80-83.

27. Castroviejo R. "Electro-Keratotome for the dissection of lamellar grafts". Transactions of the American Ophthalmological Society 56 (1958): 402-408.

28. "Skin Graft Harvesting and Donor Site Selection". In. Marcia Spear (Editions.), Skin Grafts - Indications, Applications and Current Research (2011).

29. Vistnes LM. "Grafting of skin". Surgical Clinics of North America 57 (1977): 939.

30. Netscher DT., et al. "A method for optimizing skin graft healing and outcome of wounds of the penile shaft and scrotum". Annals of Plastic Surgery 31 (1993): 447.

31. Saltz R and Bowles BJ. "Reston: an alternate method of skin graft fixation (letter)". Plastic and Reconstructive Surgery 99 (1997): 601.

32. Caldwell RK., et al. "Use of foam bolsters for securing facial skin grafts". Ear, Nose and Throat Journal 77 (1998): 490.

33. Balakrishnan C. "Dressing for skin grafts of the penis (letter)". Plastic and Reconstructive Surgery 95 (1995): 208.

34. Kromka W., et al. "Tie-over bolster dressings vs basting sutures for the closure of full-thickness skin grafts: A review of the literature". Journal of Cutaneous Medicine and Surgery 22 (2018): 602-606.
35. Akhavani MA., et al. "Quilting of full thickness grafts in the hand". Journal of Plastic, Reconstructive and Aesthetic Surgery 63.9 (2010): 1534-1537.

36. Dhillon M., et al. "A comparison of skin graft success in the head and neck with and without the use of a pressure dressing". Journal of Oral and Maxillofacial Surgery 14.2 (2015): 240-242.

37. Hallock GG. "Expanded applications for octyl-2- cyanoacrylate as a tissue adhesive". Annals of Plastic Surgery 46 (2001): 185.

38. Kilic A and Ozdengil E. "Skin graft fixation by applying cyanoacrylate without any complication (letter)". Plastic and Reconstructive Surgery 110 (2002): 370.

39. Schneider AM., et al. "A new and reliable method of securing skin grafts to the difficult recipient bed". Plastic and Reconstructive Surgery 102 (1998): 1195.

40. Hynes PJ., et al. "Split-thickness skin grafts and negative-pressure dressings in the treatment of axillary hidradenitis suppurativa". British Journal of Plastic Surgery 55 (2002): 507.

41. Wallenberg L. "Effect of early mobilisation after skin grafting to lower limbs". Scandinavian Journal of Plastic and Reconstructive and Hand Surgery 33 (1999): 411.

42. Scherer LA., et al. "The vacuum assisted closure device. A method of securing skin grafts and improving graft survival". Archives of Surgery 137 (2002): 930.

43. Clemmesen T. "The early circulation in split skin grafts". Acta Chirurgica Scandinavica 124 (1962): 11.

44. Clemmesen T. "Experimental studies on the healing of free skin autografts". Danish Medical Bulletin 14.11 (1967).

45. Converse JM., et al. "Plasmatic circulation" in skin grafts". Plastic and Reconstructive Surgery 43 (1969): 495.

46. Converse JM., et al. "Inosculation of vessels of skin graft and host bed: a fortuitous encounter". British Journal of Plastic Surgery 28 (1975): 274.

47. Peer LA and Walker JC. "The behaviour of autogenous human tissue grafts, I". Plastic and Reconstructive Surgery 7 (1951): 6.

48. Peer LA. "Transplantation of Tissues". Baltimore, Williams and Wilkins 2 (1955).

49. Birch J and Branemark PI. "The vascularization of a free fullthickness skin graft. I. A vital microscopic study". Scandinavian Journal of Plastic and Reconstructive Surgery 3 (1969): 1. 
50. Birch J., et al. "The vascularization of a free full-thickness skin graft. II. A microangiographic study". Scandinavian Journal of Plastic and Reconstructive Surgery 3 (1969): 11.

51. Birch J., et al. "The vascularization of a free full-thickness skin graft. III. An infrared thermographic study". Scandinavian Journal of Plastic and Reconstructive Surgery 3 (1969): 18.

52. Psillakis JM., et al. "Water and electrolyte changes in autogenous skin grafts. Discussion of the so-called "plasmatic circulation". Plastic and Reconstructive Surgery 43 (1969): 500.

53. Zoltan J. "Transplantationslehre". In: Gohrbandt-GabkaBerndorfer (eds), Handbuch der Plastischen Chirurgie, Band 1, Lieferung 3, Beitrag 12. Berlin, Walter de Gruyter (1965).

Volume 5 Issue 12 December 2021

(C) All rights are reserved by Sundaram Rajaram and Gauthami Sundar. 